\title{
Electron temperature difference between the o-point and x-point of
}

\section{magnetic island}

\author{
Jinhong Yang ${ }^{1,2}$, Qingquan $\mathrm{Yu}^{3}$, Sizheng Zhu ${ }^{1,2}$ and G. Zhuang ${ }^{4}$ \\ ${ }^{1}$ Institute of Plasma Physics, Chinese Academy of Sciences, Hefei, 230031, China \\ ${ }^{2}$ Center for Magnetic Fusion Theory, Chinese Academy of Sciences, Hefei, 230031, \\ China \\ ${ }^{3}$ Max-Planck-Institute für Plasmaphysik, EURATOM Association, 85748, Garching, \\ Germany \\ ${ }^{4}$ College of Electrical and Electronic Engineering, Huazhong University of Science and \\ Technology, Wuhan, 430074, China \\ E-mail: jhyang@ipp.ac.cn
}

The electron temperature difference between the o-point and the x-point of magnetic island is studied numerically by solving the two-dimensional energy transport equation. It is found that, even without a localized radio-frequency heating at the island's o-point, there is usually a temperature difference between these two points. This difference depends on the radial profile of the heating power deposition, the ratio between the parallel and the perpendicular heat conductivity and the island width, and it takes a minimum when the island width is about twice the local heat diffusion layer width. The effect of the temperature difference on the island growth is further studied, and the peaked heating power density profile at magnetic axis is found be destabilizing. 


\section{I . Introduction}

Magnetic islands are often observed in tokamak plasma experiments. These islands can be driven by an unfavorable plasma current density gradient (a positive tearing

mode stability index $\Delta^{\prime}$ ), the perturbed bootstrap current (neoclassical tearing modes), or the electron temperature gradient (drift tearing mode) [1-7]. The heat transport across the island is an important issue of the magnetic island physics. It is well known that for a sufficiently large island, the electron temperature profile becomes nearly flat in the island region due to the fast parallel transport. Such a locally flat temperature (and the plasma pressure) profile leads to the perturbed bootstrap current driving the neoclassical tearing modes [2-6]. On the other hand, a local flat temperature profile results in a global reduction of the electron temperature (and the plasma pressure) from the island up to the magnetic axis [2-6], which have been found to limit the plasma pressure or even cause disruptions in tokamak experiments [2-6, 8].

The change of the electron temperature by the magnetic island has been studied in

the limits of $w<<w_{c}$ and $w>>w_{c}[9,10]$, where $w$ is the island width, and $w_{c}=a\left(\chi_{\perp} / \chi_{/ /}\right)^{1 / 4}\left(\varepsilon a n / 8 L_{q}\right)^{-1 / 2}$ is the heat diffusive layer width at the rational surface, $\chi_{\|}$and $\chi_{\perp}$ are the parallel and perpendicular heat difusivities, $L_{q}=q / q^{\prime}, q$ 
is the safety factor, a is the plasma minor radius, $\varepsilon=a / R$ is the inverse aspect ratio,

and ${ }^{n}$ is the toroidal mode number $[9,10]$. For ${ }^{w}<<w_{c}$, the contribution to the radial transport from the parallel transport along the magnetic field lines is smaller than that from the perpendicular transport. While for $w>>w_{c}$, the electron temperature profile becomes nearly flat in the island region except in a thin layer around the island's separatrix $[9,10]$. In these studies, the effect of the heat source or sink in the island region is neglected.

In this paper, the electron energy transport across the magnetic island is studied numerically by solving the two-dimensional transport equation. Different heat source (or sink) profiles are taken into account. We focus on the temperature difference between the o-point and x-point of the magnetic island, as this difference leads to the difference in the plasma resistivity and therefore a corresponding difference in the plasma current density, which affects the island width at nonlinear saturation. It is well known that a higher electron temperature or a lower plasma resistivity at the island's o-point is stabilizing for the island, leading to the method of localized RF heating at the island's o-point for stabilizing the island $[6,11,12]$. It will be shown in this paper that, even when there is no localized RF heating in the island region, there is usually a difference in the electron temperature between the o-point and x-point of the magnetic island, and such a difference depends on the radial profile of the heat source (or sink) and affects the stability of the island. 


\section{II . MODEL}

\section{A. Computational model}

The periodic cylindric geometry is utilized. The magnetic field $\stackrel{\vec{B}}{\text { is defined as }}$

$$
\stackrel{\nu}{B}=B_{0 t} \stackrel{\mathscr{B}_{t}}{e^{\prime}}-\left(n_{1} r / m_{1} R\right) B_{0 \theta} \stackrel{\boldsymbol{B}_{\theta}}{e_{\theta}}+\nabla \psi \times \stackrel{B}{e_{t}}
$$

Where $\psi$ is the helical magnetic flux function, $m / r$ and $n / R$ are the wave vectors in

$\stackrel{e}{e}_{\theta}$ (poloidal) and $\stackrel{e}{e}$ (toroidal) direction respectively, the subscript 0 denotes the equilibrium quantity, and $R$ is the major radius.

The radial profile of the safety factor $q(r)=r B_{0 t} /\left(R B_{0 \theta}\right)$ is chosen to be the form

$$
q(r)=q_{0} e^{r / L_{q}}
$$

to have a constant magnetic shear along the minor radius, where $q_{0}=0.45$ and

$L_{q}=0.5 a$ are taken to ensure $q=3 / 2$ surface to be inside the plasma $(0 \leq r \leq a)$.

The perturbed helical magnetic flux is taken to be

$$
\psi_{i}(r)=\psi_{i 0}(r / a)^{2}(1-r / a)^{2}
$$

to have a smooth change along the minor radius except mentioned elsewhere.

The following electron energy transport equation 


$$
\frac{3}{2} n_{e} \frac{\partial T_{e}}{\partial t}=n_{e} \nabla \cdot\left(\chi_{/ /} \nabla_{/ /} T_{e}\right)+n_{e} \nabla \cdot\left(\chi_{\perp} \nabla_{\perp} T_{e}\right)+p(r)
$$

is solved, where, $T_{e}, n_{e}$, and $p(r)$ are electron temperature, electron density, and the heating power density, respectively. Here only the parallel and the perpendicular heat diffusion are considered, and the convective transport is neglected. Furthermore, $n_{e}$, $\chi_{/ /}$and $\chi_{\perp}$ are assumed to be constant across the whole minor radius for simplicity. The radial profile of the equilibrium electron temperature in steady state is therefore determined by the heat source $\mathrm{p}(\mathrm{r})$ in Eq. (4). In our calculations the same q-profile is utilized for different equilibrium temperature profiles, so that the effect of plasma resistivity on the plasma current density profile is neglected. We only focus on the heat transport with given equilibrium and magnetic perturbations in this paper, which is characterized by the parameter $w / w_{c}$ according to Refs. [9] and [10].

$\psi$ and $T_{e}$ are expressed in terms of Fourier components of the form $A=A_{0}(r)+\sum A_{j}(r) \cos \left(m_{j} \theta+n_{j} \varphi\right)$, where $m_{j}$ and $n_{j}$ are the poloidal and toroidal mode numbers of the $j$ th component, and $\theta$ and $\phi$ are the poloidal and toroidal angle respectively. 
The heat source $p(r)$ in Eq. (4) is taken to be the form

$$
p(r)=p_{0}\left(1-r^{\alpha}\right)^{\beta}
$$

In Fig. 1 different radial profiles of the heating power density $p$ are shown, obtained with different value of $\alpha$ and $\beta$. The total heating power $\left(P=\int_{0}^{a} 2 \pi r p(r) d r\right)$ is the same for all these curves.

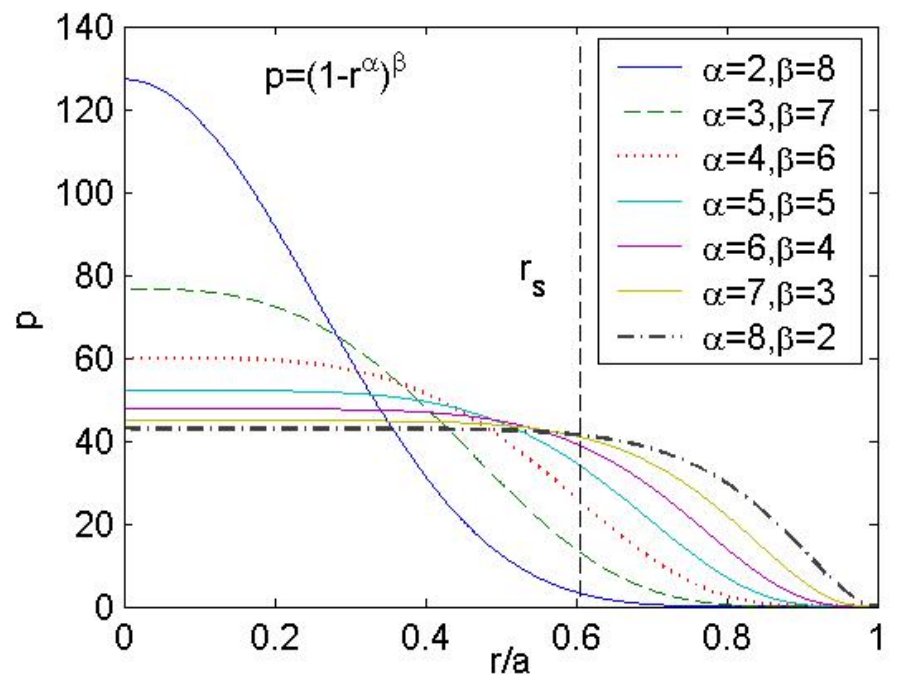

Fig.1

The boundary conditions are $T_{e}(r=a)=T_{e 0}(r=a)=0$ and $\frac{d T_{e}(r=0)}{d r}=0$, where

$T_{e 0}$ is the equilibrium electron temperature obtained without the magnetic island. The length is normalized to $a$, the magnetic field to $B_{0 t}$, and $T_{e}$ to $T_{e 0}(r=0)$.

\section{B. Effect of temperature perturbation on magnetic island growth}


The island growth can be obtained from perturbed Ohm's law $[8,9]$

$$
\frac{d \psi}{d t}=(\eta j)_{1} \approx \eta j_{1}+\eta_{1} j
$$

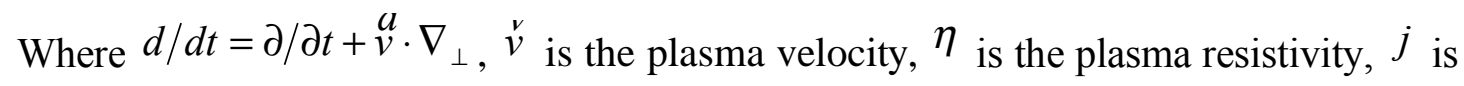
the plasma current density, and the subscript 1 denotes perturbed quantity.

Since $j_{1}=\nabla^{2} \psi, j=\frac{d\left(r B_{\theta}\right)}{d r}, \eta_{1}=-\frac{3}{2} \frac{T_{e(o)}-T_{e(x)}}{T_{e(x)}} \eta$, and $\psi=\frac{w^{2}}{16} \frac{q^{\prime}}{q} B_{0 \theta}$, one finds from Eq. (6) that $[8,9]$

$$
\frac{d w}{d t} \approx \eta\left(\Delta^{\prime}+0.5 \Delta_{\delta T}\right)
$$

Where $\Delta^{\prime}=\left.\frac{\psi^{\prime}}{\psi}\right|_{r_{s}-w / 2} ^{r_{s}+w / 2}$ is the conventional tearing mode stability index, $r_{s}$ is the radial location of the rational surface, $\Delta_{\delta T}=C_{1} / w$ is due to the temperature difference between the island's o-point and x-point, $C_{1}=24\left(1-\frac{2}{r_{s}} \frac{q}{q^{\prime}}\right) \delta T_{e}$, and the parameter $\delta T_{e} \equiv \frac{T_{e(o)}-T_{e(x)}}{T_{e(x)}}$ defines the relative temperature difference between the island's opoint and x-point. $T_{e(o)}$ and $T_{e(x)}$ are the electron temperature at magnetic island's opoint and x-point, respectively. 


\section{III . Modeling results}

A single $m / n=3 / 2$ magnetic island is considered here. The numerical calculation of the heat transport across magnetic islands is usually quite challenging for a high ratio of $\chi_{/ /} / \chi_{\perp}$, because the pollution by numerical errors can easily swamp the true perpendicular transport. Our calculations are based on a new numerical method, which has been shown to suppress this artificial perpendicular heat flux[13].

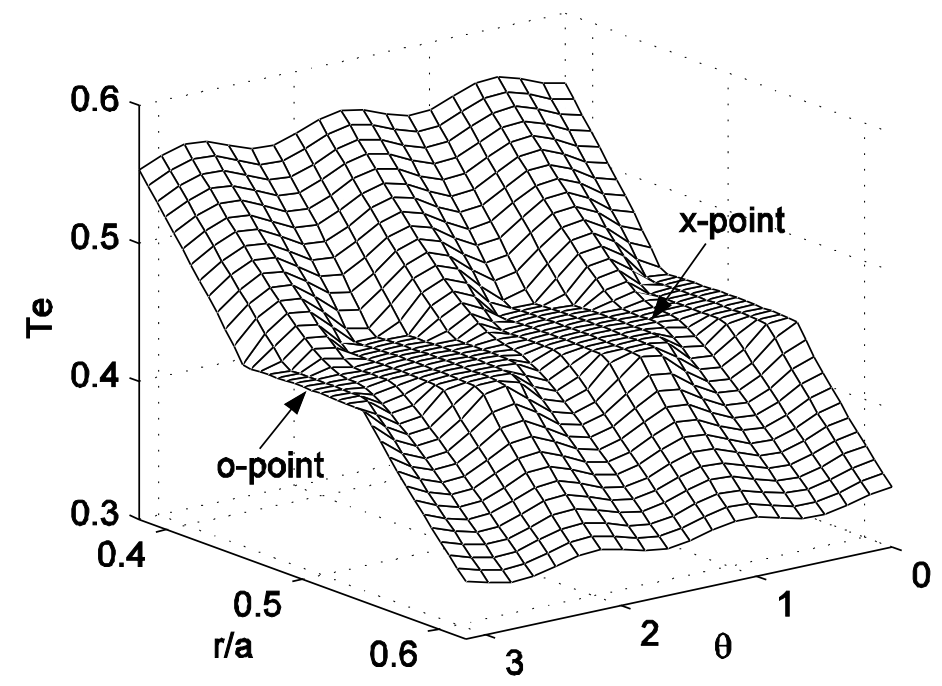

Fig.2

The three-dimension plot of the electron temperature $T_{e}$ in the presence of a single

$m / n=3 / 2$ island, obtained from Eq. (4), is shown in Fig.2, with $\alpha=2$ and $\beta=8$. 
The $q=3 / 2$ surface is at $r_{3 / 2}=0.601 a$, the island width is $w_{3 / 2}=0.082 a$, and $\chi_{/ /} / \chi_{\perp}=10^{9}$ is taken. It is seen that $T_{e}$ becomes flat in the island region due to a large island width and a high ratio of $\chi_{/ /} / \chi_{\perp}$, being similar to previous results $[9,10]$.

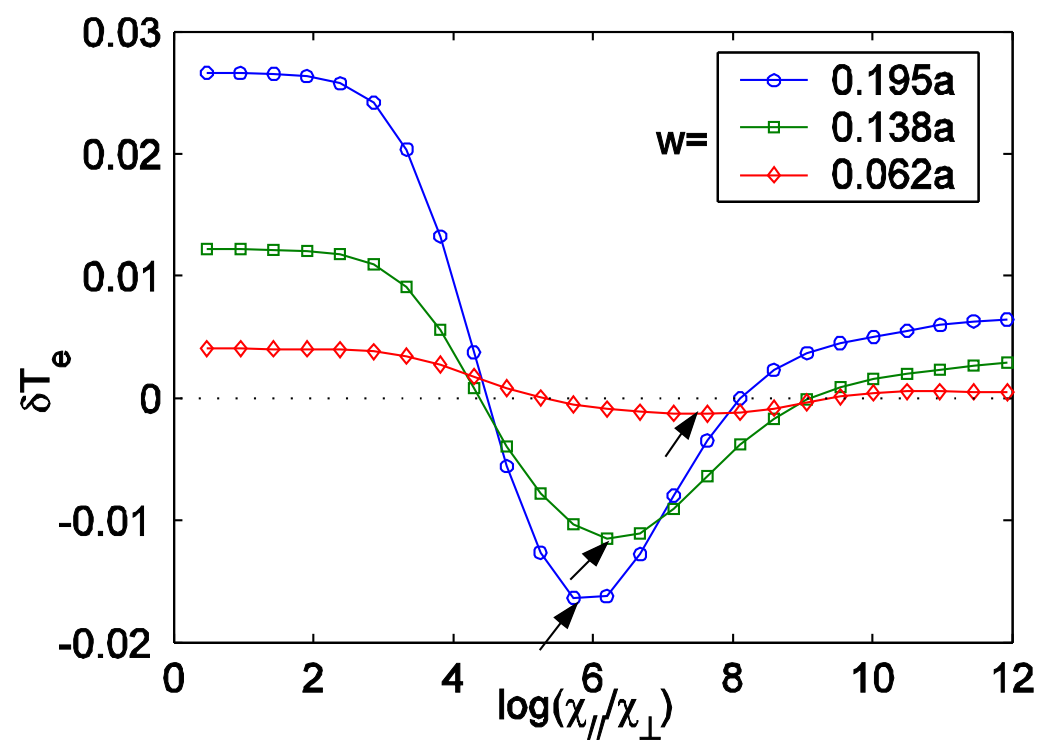

Fig.3

When looking carefully into the temperature profile inside the island, however, one finds that usually there is a difference in the electron temperature between the island's o-point and x-point. In fig. 3 the quantity $\delta T_{e}$ as a function of $\log \left(\chi_{/ /} / \chi_{\perp}\right)$ is shown for $w / a=0.195,0.138$ and 0.062 , with other parameters being the same as those of Fig.

2. It is seen that $\delta T_{e}$ is positive for a sufficiently low $\chi_{/ /} / \chi_{\perp}$. This is caused by the inwards (outwards) shift of the island's o-point (x-point) from the resonant surface due 
to a negative radial gradient of helical flux perturbation at the rational surface given by

Eq. (3), and the parallel transport is not important for a sufficiently low $\chi_{/ /} / \chi_{\perp}$. For a

sufficiently large $\chi_{/ /} / \chi_{\perp}, \delta T_{e}$ is also positive, as in this case the temperature is a constant on the magnetic surface both inside and outside the island except in a thin layer around the island separatix [9], and the finite heat source inside the island leads to a higher temperature at the island's o-point than that at the x-point. For a intermediate value of $\chi_{/ /} / \chi_{\perp}$, however, $\delta T_{e}$ takes a negative value and has a minimum $\delta T_{\min }$ ( as indicated by black arrows ). These minimum decreases for a larger island but located at different value of $\chi_{/ /} / \chi_{\perp}$.

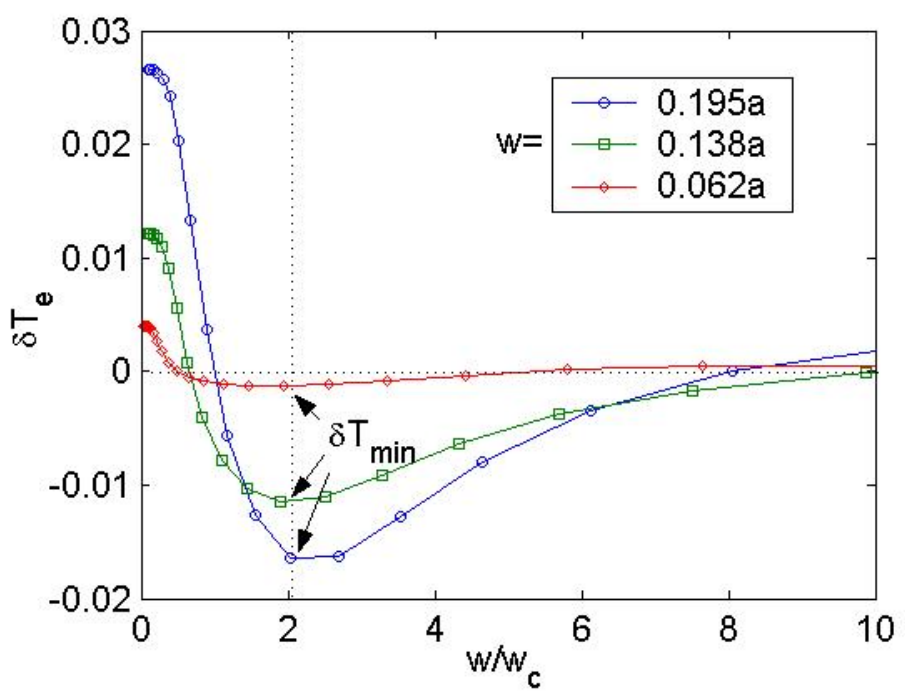

Fig.4

Corresponding to Fig.3, in Fig.4 the value $\delta T_{e}$ is plotted as a function of $w / w_{c}$. It 
is seen that, all these minimum values of $\delta T_{e}$ are approximately at $w \approx 2 w_{c}$. The contribution from the parallel transport to the radial transport becomes comparable to that from the perpendicular one for $w \sim w_{c}$. In this case the temperature is not constant on the magnetic surface. Part of heating power in the central region between the magnetic axis and the island transports outwards along the field lines and pass through the island region. As the field line length is much longer near the x-point than elsewhere in the island region, a higher temperature difference between the x-point and the outer edge of the island at $r>r_{s}$ is required for maintaining the outwards parallel heat flux. This leads to a higher temperature at the x-pint than that at the island's o-point if the local heating power inside the island is not significant. On the other hand, the parallel temperature gradient decreases due to the parallel heat flux with increasing $\chi_{/ /} / \chi_{\perp}$, which in turn keeps the parallel heat flux to be finite, as the heating power in the central region is finite. Eventually, for a sufficiently large $\chi_{/ /} / \chi_{\perp}$ the electron temperature approaches a constant on the magnetic surface being very close to the separatrix [9], and the electron temperature at the o-point becomes higher than that at the x-point if the heat source inside the island is positive 


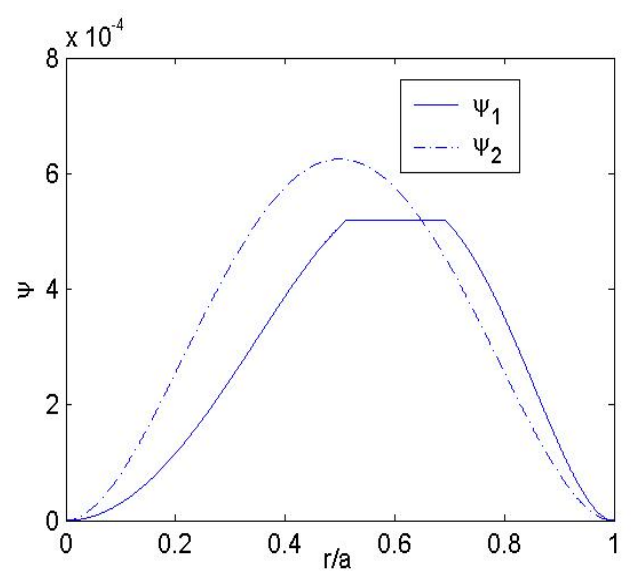

Fig.4a

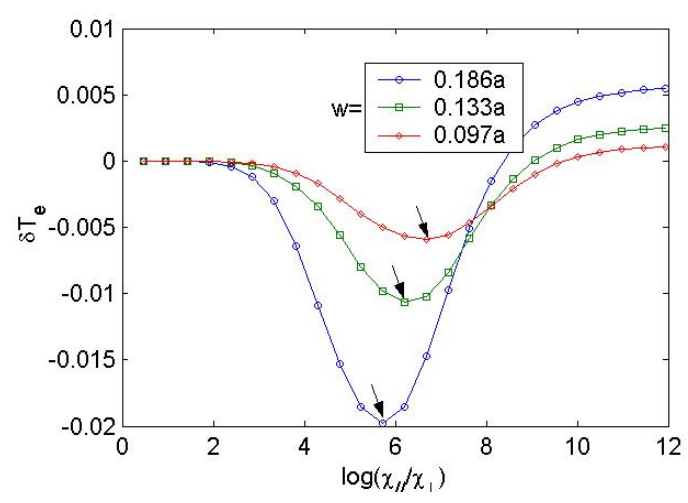

Fig.4b

When the helical flux perturbation is taken to be $\psi_{1}=\psi_{i 0} r^{2}\left(1-r^{2.5}\right)^{2}$ outside the

island but to be a constant across the island region, as shown by $\psi_{1}$ in Fig. 4 a ( $\psi_{2}$ in Fig. 4a shows the radial profile of the helical flux perturbation given by Eq. (3)), the corresponding $\delta T_{e}$ as a function of $\log \left(\chi_{/ /} / \chi_{\perp}\right)$ is shown in Fig. $4 \mathrm{~b}$ for $w / a=0.186$, 0.133 and 0.097 , with other parameters being the same as those of Fig. 3. For a constant- $\psi$ in the island region there is no radial separation of the o- and x-points, so 
that $\delta T_{e}$ approaches zero for $\operatorname{low} \log \left(\chi_{/ /} / \chi_{\perp}\right)$ as shown in Fig. $4 \mathrm{~b}$ as expected.

Comparing Fig. 3 and $4 \mathrm{~b}$ one finds that, the minimum value of $\delta T_{e}$ for both cases is located at $w \approx 2 w_{c}$, and the $\delta T_{e}$ in Fig. $4 \mathrm{~b}$ is similar to that in Fig. 3 in the large $\log \left(\chi_{/ /} / \chi_{\perp}\right)$ region. The major difference between Figs. 3 and $4 \mathrm{~b}$ is in the low $\log \left(\chi_{/ /} / \chi_{\perp}\right)$ region.

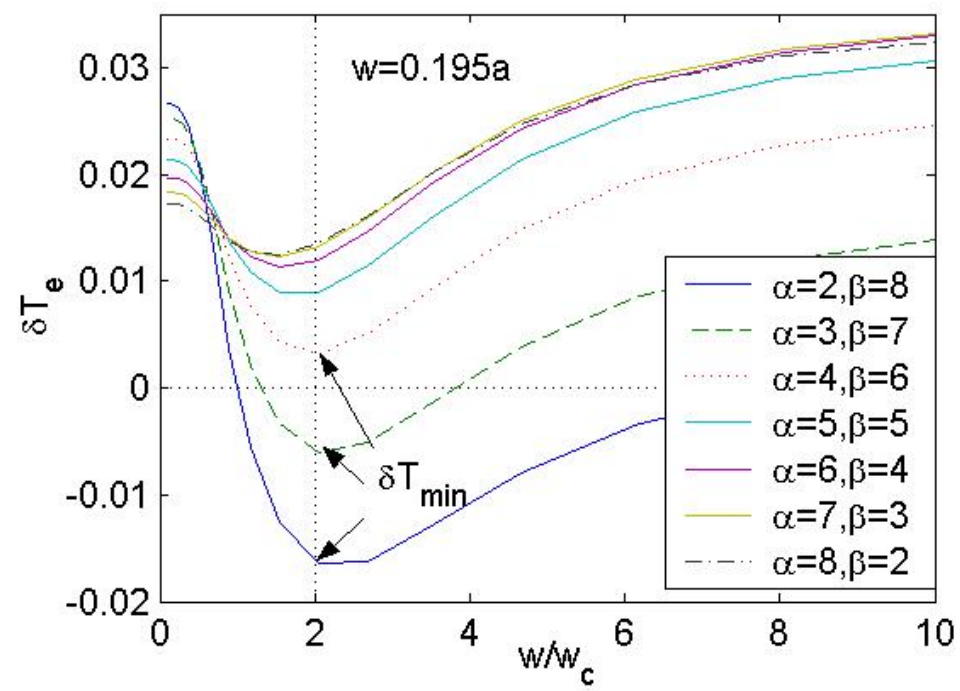

Fig.5

For other profiles of heat source as shown in fig.1, the value of $\delta T_{e}$ as a function of $w / w_{c}$ is shown in fig.5. When the heat source becomes less peaking at the magnetic 
axis, the value of $\delta T_{\min }$ increase from negative to positive value due to more heating power inside the island, but the minimum of $\delta T_{e}$ remains at $w \sim w_{c}$.

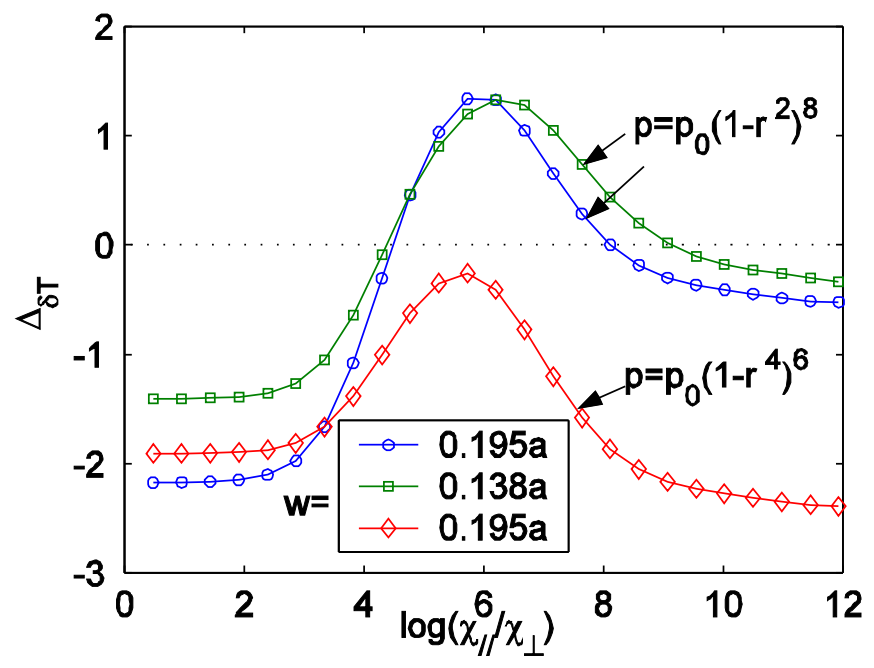

Fig.6

The $\delta T_{e}$ affects the island growth as indicated by the term $\left(\Delta_{\delta T}\right)$ in Eq.(7). In

Fig.6, the value of $\Delta_{\delta T}$ as a function of $\log \left(\chi_{/ /} / \chi_{\perp}\right)$ is shown for $p(r)=p_{0}\left(1-r^{2}\right)^{8}$

by the curves with circles and squares and for $p(r)=p_{0}\left(1-r^{4}\right)^{6}$ by the curve with diamonds. Other parameters are the same as those of Fig.3, and $q / q^{\prime}=L_{q}=0.5 a$ is assumed, which leads to $C_{1}=-15.9 \delta T_{e}$. It is seen from the curves with circles and 
squares that, the value of $\Delta_{\delta T}$ is positive for $4<\log \left(\chi_{/ /} / \chi_{\perp}\right)<8$, indicating a destabilizing effect on the island in this case. The curve with diamonds shows that $\Delta_{\delta T}$ is negative for all values of $\chi_{/ /} / \chi_{\perp}$, so that more heating power at the island region is stabilizing.
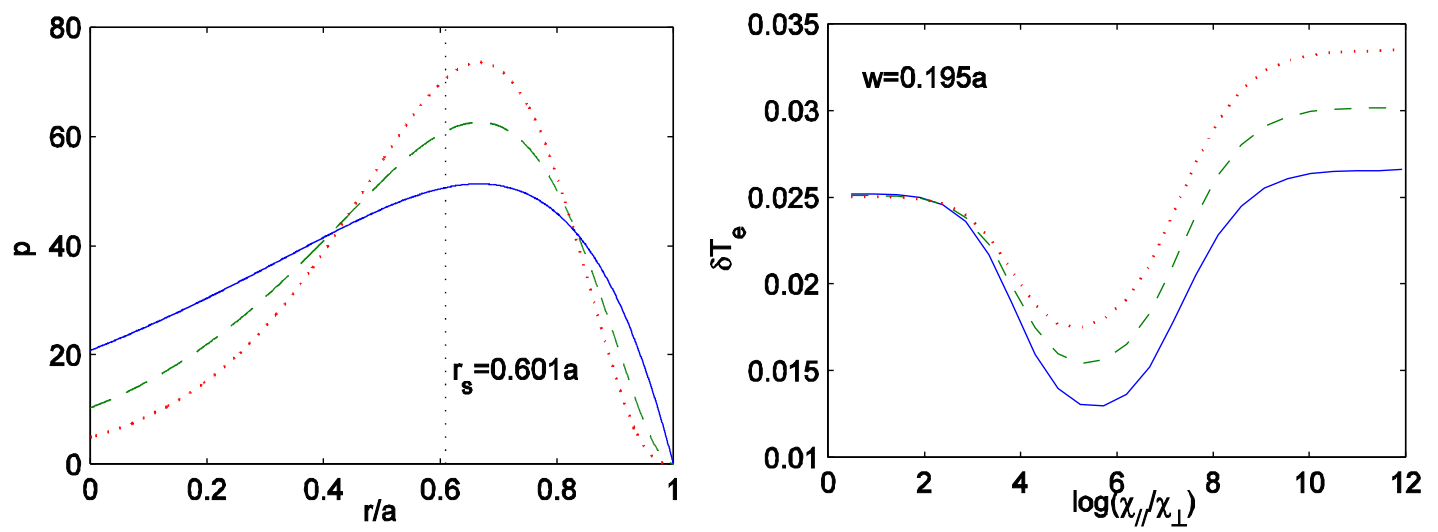

Fig.7

Fig.8

In tokamak experiments, depending on the heating method and the plasma parameters, the heat source can also be off-axis peaked, as shown in Fig.7. The total amount of the heating power is the same for these cases. Corresponding to fig.7, the values of $\delta T_{e}$ as a function of $\log \left(\chi_{/ /} / \chi_{\perp}\right)$ is shown in fig.8. The island width is $w / a=0.195$, and the other parameters are the same as those of Fig.3. It is seen that, more heating power deposited in the island region leads to a larger value of $\delta T_{e}$, which has a stabilizing effect on the magnetic island, as seen in the experiments [11]. This 
case is in fact similar to the localized continuous RF heating in the island region [11].
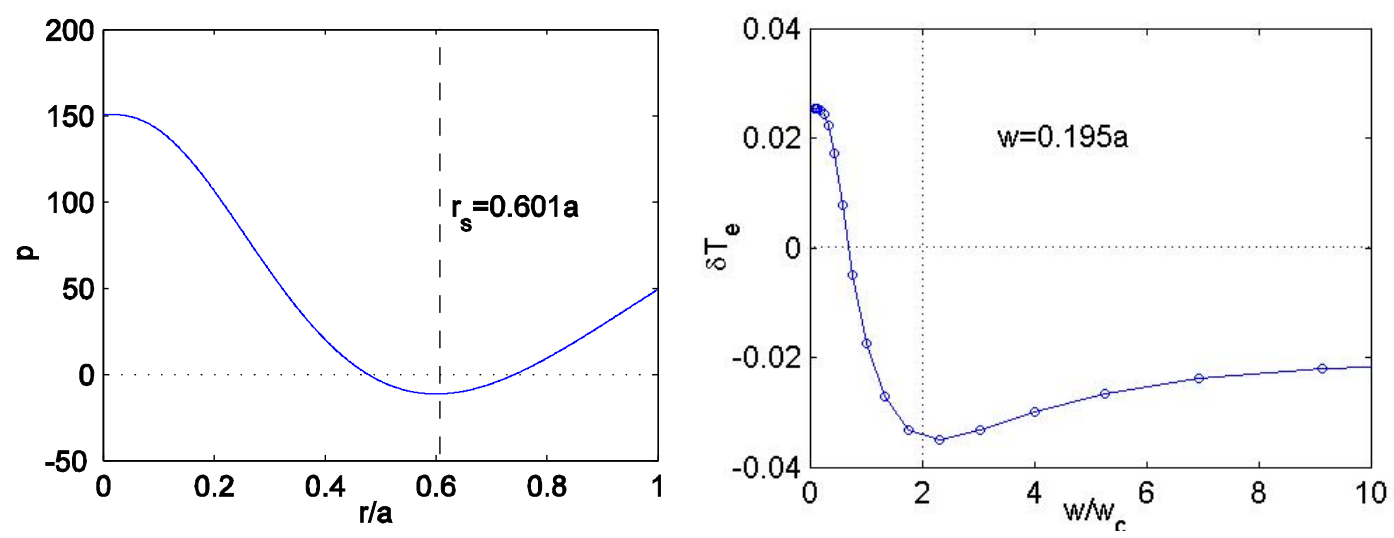

Fig.9

Fig.10

In high density tokamak discharges it is often found that, when there is a large island, strong impurity radiation occurs in the island region [15-17]. This corresponds to a negative local heat source in the magnetic island region when the local radiation power density exceeds the heating power density. Such a case is modeled by the heat source profile as shown in Fig.9, with a negative local heat source in island region.

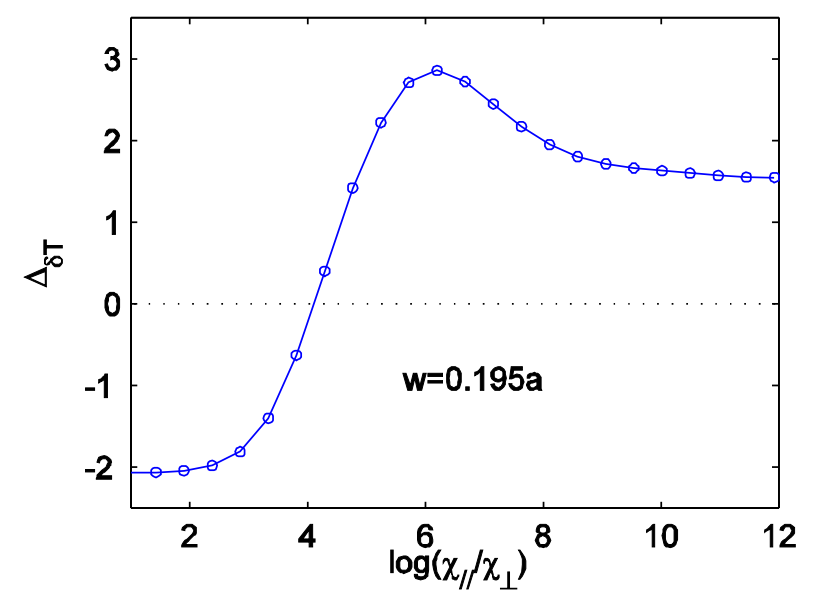

Fig.11

Corresponding to fig.9, in fig.10 $\delta T_{e}$ as a function of $w / w_{c}$ is shown. It is seen 
that the minimum value of $\delta T_{\min }$ also appears at $w \approx 2 w_{c}$, and the absolute value of $\delta T_{e}$ increase to $\delta T_{\min } \sim 4 \%$. In fig. 11, the value of $\Delta_{\delta T}$ as a function of $\log \left(\chi_{/ /} / \chi_{\perp}\right)$ is shown. The values of $\Delta_{\delta T}$ are positive in the region of $\log \left(\chi_{/ /} / \chi_{\perp}\right)>4$, indicating that a local negative heat source in the island region is destabilizing, in agreement with the experimental observations on ASDEX UPGRADE [15]. The value of $\Delta_{\delta T}$ is of the same order as the low-m tearing mode stability index $\Delta^{\prime}[6]$, one usually has $\Delta^{\prime} \sim-m / r_{s}[6,18]$.

\section{IV . Discussion and Summary}

It is well known that, with continuous increase plasma density, plasma will be eventually subjected to the density limit and disrupted [8]. Before the major disruption, the growth of the $m=2$ magnetic island is observed, followed by a fast drop in the plasma temperature and a slower decay of the plasma current. The growth of the $m=2$ magnetic island is usually thought to be caused by the steep of the plasma current density gradient as a result of cool of the outer regions of the plasma by impurities or plasma recycling in high density discharges $[8,15-17,19]$. In high density plasmas the 
ohmic heating power is mainly in the central region where the plasma temperature and the current density are high. It is seen from Figs. 3 and 6 that such a peaked power deposition profile can lead to a lower electron temperature at the island's o-point than that at the x-point, which could play a role in enhancing the island growth for $w \sim w_{c}$, in addition to the steep of the plasma current density gradient. If the local impurities radiation power exceeds the local heating power in the island region, the destabilizing effect is larger as seen from Fig. 11. For tokamak plasmas with $n=1 L_{q}=a R / a=3$ $\chi_{\perp}=1 \mathrm{~m}^{2} / \mathrm{s} \quad T_{e}=300 \mathrm{ev}$ and $n_{e}=5 \times 10^{19} \mathrm{~m}^{-3}$ at the $q=2$ surface, one has $w_{c}=0.035 a$ by using the classical parallel heat conductivity. With the increase of island width, the electron temperature decreases [8], leading to a larger value of $w_{c}$, as $w_{c} \sim\left(\chi_{\perp} / \chi_{/ /}\right)^{1 / 4} \sim T_{e}^{-5 / 8} n_{e}^{1 / 4} \chi_{\perp}^{1 / 4}$. The increase of $w_{c}$ would be slower than that of the island width if one assumes that the cool down of the $q=2$ region is linearly proportional to the island width (of course, this depends on the radial temperature gradient outside the $q=2$ surface), and that $\chi_{\perp}$ does not significantly change. It is 
seen from Figs. 9-11 that, once $w>2 w_{c}$ and the local radiation power exceeds the local heating power in the island region, the mechanism studied here can play a role in the nonlinear growth of the island in high density discharges [8, 15-17, 19].

The net heat source profile due to both the heating power and the plasma radiation depends on the heating method and plasma parameters. In high density tokamak discharges strong impurity radiation is in the plasma edge and the $q=2$ region $[15-17,19]$. The radiation power can be of the same order or even larger than the heating power $[8,15,19]$, suggesting a much negative heat source in the island region than that shown in Fig. 9 and therefore a more negative $\Delta_{\delta T}$ than that shown in Fig. 11. While with localized RF heating in the island region, the net heat source profile is similar to that shown in Fig.7 or becomes even more peaked at the rational surface, depending on the RF power. This corresponds to a stabilizing effect as seen in the experiments [11].

Future studies using self-consistent radial profiles of the plasma current density and the electron temperature, including the convective transport and simultaneously calculating both the magnetic and temperature perturbations are still required to further study the effect of $\delta T_{e}$ on the island growth.

In summary, the electron temperature difference between the island's o-point and $\mathrm{x}$-point is investigated by numerical modeling. We find that, 
1. When $w \approx 2 w_{c}$, the value of $\delta T_{e}$ has a minimum value $\delta T_{\min }$.

2. The value of $\delta T_{\min }$ depends on the heat source profile. For peaked on-axis heating power density profile, $\delta T_{\min }$ is negative. When the local heat source in the island region is negative, as in the case that the local radiation power exceeds the local heating power, the negative value region of $\delta T_{e}$ extends to higher $\chi_{/ /} / \chi_{\perp}$. With more heating power deposited in the island region, $\delta T_{\min }$ becomes positive as expected.

3. The value of $\delta T_{e}$ affects the nonlinear saturated island width, as it can lead to a stabilizing (or destabilizing) effect being comparable to that of the low-m tearing mode stability index $\Delta^{\prime}$. This effect is determined by the local power density in the island region for a large magnetic island with $w>>w_{c}$.

\section{ACKNOWLEDGEMENT}

This work is supported by National Natural Science Foundation of China (No. 10675192) and the Chang-Jiang scholarship project of the Ministry of Education of China. The authors would like to thank the Center for Computational Science, Hefei Institute of Physical Sciences for its support. 


\section{References}

[1] H.P.Furth, J.Killen, and M.N.Rosenbluth, Phy.Fluids 6, 459(1963)

[2] Z. Chang, J. D. Callen, E. D. Fredrickson, R. V. Budny, C. C. Hegna, K. M.

McGuire, M. C. Zarnstorff, and TFTR group, Phys. Rev. Letts, 74, 4663(1995).

[3] H. Zohm, G. Gantenbein, A. Gude, S. Günter, F. Leuterer, and M. Maraschek, J. Meskat, W. Suttrop, Q. Yu, ASDEX Upgrade Team, and ECRH-Group (AUG), Phys. Plasmas 8, 2009(2001).

[4] S. Günter,A. Gude, M. Maraschek, S. Sesnic, H. Zohm, ASDEX Upgrade Team, and D. F. Howell, Phys. Rev. Letters, 87, 275001(2001).

[5] R. J. La Haye, L. L. Lao, E. J. Strait, and T. S. Taylor, Nucl. Fusion 37, 397(1997).

[6] O. Sauter, R. J. La Haye, Z. Chang, D. A. Gates, Y. Kamada, H. Zohm, A. Bondeson, D. Boucher, J. D. Callen, M. S. Chu, T. A. Gianakon, O. Gruber, R. W. Harvey, C. C. Hegna, L. L. Lao, D. A. Monticello, F. Perkins, A. Pletzer, A. H. Reiman, M. Rosenbluth, E. J. Strait, T. S. Taylor, A. D. Turnbull, F. Waelbroeck, J. C. Wesley, H. R. Wilson, and R. Yoshino, Phys. Plasmas 4, 1654(1997).

[7] Q. Yu, S. Günter, B. D. Scott, Phys. Plasmas 10, 797(2003).

[8] J.Wesson, Tokamaks Third edition, edited by J. Birman, et al. (Clarendon Press,Oxford,1987), p. 356.

[9] Richard Fitzpatrick, Phys. Plasmas 2, 825(1995)

[10] Q. Yu, Phys. Plasmas 13 (2006) 062310.

[11] E. Westerhof, A. Lazaros, E. Farshi, M.R. de Baar, M.F.M. de Bock, I.G.J. 
Classen, R.J.E. Jaspers, G.M.D. Hogeweij, H.R. Koslowski, A. Kr”amer-Flecken, Y. Liang, N.J. Lopes Cardozo and O. Zimmermann, Nucl.Fusion (2007)47, 85-90

[12] Qingquan Yu and Sibylle Gunter Plasma Phys. Control. Fusion 40(1998) $1977-1987$

[13] S.Günter Q.Yu, and K.Lackner, Journal of Computational Physics 209(2005) $354-370$

[14] Qingquan Yu and Sibylle Günter Phys. Plasmas 5, 3924(1998)

[15] W. Suttrop, K. Buchl, J.C. Fuchs, M. Kaufmann, K. Lackner, M. Maraschek, V. Mertens, R. Neu, M. Schittenhelm, M. Sokoll, H. Zhom, ASDEX Upgrade Team, Nucl.Fusion, Vol.37, 119 (1997)

[16] D. J. Ward and J.A. Wesson, Nucl. Fusion, 32, 1117 (1992)

[17] J.A. Wesson, R.D. Gill, M. Hugon, F.C.Schuller, J.A.Snipes, D.J.Ward, D.V.Bartlett, D.J.Campbell, P.A.Duperrex, A.W.Edwards, R.S.Granetz, N.A.O.Gopttardi, T.C.Hender, E.Lazzaro, P.J.Lomas，N.Lopes cardozo，K.F.Mast, M.F.F.Nave, N.A.Salmon, P.Smeulders, P.R.Thomas, B.J.D.Tubbing, M.F.Turner, A.Weller, Nucl. Fusion, 29, 641 (1989)

[18] Q. Yu, S. Günter and K. Lackner, Phys. Plasmas 11, 140(2004)

[19] F.C. Schüller Plasma Phys. Control. Fusion 37 (1995) A125 


\section{Caption}

FIG. 1(color online) Different radial profiles of the heating power density $p$ are shown, obtained with different value of $\alpha$ and $\beta$ in Eq. (5). The total heating power ( $\left.P=\int_{0}^{a} 2 \pi r p(r) d r\right)$ is the same for all these curves.

FIG. 2(color online) Three dimensional plot of the electron temperature $T_{e}$ in the presence of a single $m / n=3 / 2$ island, obtained with $\alpha=2$ and $\beta=8$.

Fig.3(color online) $\quad \delta T_{e}$ versus $\log \left(\chi_{/ /} / \chi_{\perp}\right)$ for $w / a=0.195,0.138$ and 0.062 , with other parameters being the same as those of Fig. 2. For a intermediate value of $\chi_{/ /} / \chi_{\perp}, \delta T_{e}$ takes a minimum ( as indicated by black arrows ).

Fig.4(color online) Same as Fig. 3, but $\delta T_{e}$ is shown as a function of $w / w_{c}$. All these minimum values of $\delta T_{e}$ are approximately at $w / w_{c} \sim 2$.

Fig.4a(color online) Radial profiles of the $\mathrm{m} / \mathrm{n}=3 / 2$ component of helical flux perturbations. $\psi_{1}$ is taken to be $\psi_{1}=\psi_{1} r^{2}\left(1-r^{2.5}\right)^{2}$ outside the island but to be a constant across the island region, while $\psi_{2}$ is given by Eq. (3). 
Fig.4b(color online) $\delta T_{e}$ versus $\log \left(\chi_{/ /} / \chi_{\perp}\right)$ for $w / a=0.186,0.133$ and 0.097 ,

with the helical flux perturbation given by the $\psi_{1}$ in Fig. 4a and other parameters being

the same as those of Fig. 3. $\delta T_{e}$ is zero for a sufficiently low $\chi_{/ /} / \chi_{\perp}$ due to a constant$\psi$ in the island region.

Fig. 5(color online) $\delta T_{e}$ versus $w / w_{c}$ for other radial profiles of heat source in fig.1.

When the heat source becomes less peaking at the magnetic axis, $\delta T_{\min }$ increase from negative to positive value due to more heating power inside the island, but the minimum of $\delta T_{e}$ remains at $w \approx 2 w_{c}$.

Fig.6(color online) $\quad \Delta_{\delta T}$ versus $\log \left(\chi_{/ /} / \chi_{\perp}\right)$ for $p(r)=p_{0}\left(1-r^{2}\right)^{8}$ by the curves with circles and squares and for $p(r)=p_{0}\left(1-r^{4}\right)^{6}$ by the curve with diamonds. Other parameters are the same as those of Fig.3, and $q / q^{\prime}=L_{q}=0.5 a$ is assumed, which leads to $C_{1}=-15.9 \delta T_{e}$.

Fig. 7(color online) Radial profiles of off-axis peaked heating power density. The total amount of the heating power is the same for these cases. 
Fig. 8 (color online) Corresponding to fig. $7, \delta T_{e}$ versus $\log \left(\chi_{/ /} / \chi_{\perp}\right)$. The island width is $w / a=0.195$, and the other parameters are the same as those of Fig.3. More heating power deposited in the island region leads to a larger value of $\delta T_{e}$. Fig. 9(color online) The radial profile of the heating power density, being negative in the magnetic island region due to the assumption that the local radiation power density exceeds the heating power density.

Fig. 10(color online) Corresponding to fig.9, $\delta T_{e}$ versus $w / w_{c} . \delta T_{\min }$ appears at $w \approx 2 w_{c}$, and the absolute value of $\delta T_{e}$ increase to $\delta T_{\min } \sim 4 \%$.

Fig. 11 (color online) Corresponding to fig.9, the value of $\Delta_{\delta T}$ versus $\log \left(\chi_{/ /} / \chi_{\perp}\right)$. The values of $\Delta_{\delta T}$ are positive in the region of $\log \left(\chi_{/ /} / \chi_{\perp}\right)>4$, indicating that a local negative heat source in the island region is destabilizing. 